\title{
大气复合污染条件下新粒子生成和增长机制及其环境影响
}

\author{
胡敏* 尚冬杰郭松吴志军 \\ (环境模拟与污染控制国家重点联合实验室 北京大学环境科学与工程学院 北京 100871)
}

\begin{abstract}
摘要 新粒子生成与增长是一个从分子到纳米颗粒物的微观尺度演变过程, 对区域到全球尺度空气质量和气候造成显 著影响. 总结了国内外对新粒子生成与增长物理化学机制的研究进展和局限性. 提出以揭示新粒子生成、增长及其产 生环境效应的机制为核心目标, 利用外场观测、烟雾箱模拟和模式模拟等多种手段, 开发和应用先进的研究技术, 辨识 参与并促使快速成核的关键前体物种, 弄清驱动新粒子初始和后续增长的化学和物理机制, 以揭示大气复合污染条件 下快速成核和持续增长的条件与机制并评价新粒子对大气环境的影响. 在全面理解大气复合污染条件下成核和增长机 制及其环境效应的基础上提高对区域霧形成机制的认识，也推进国际相关领域对污染大气环境新粒子生成与增长机制 普遍性和差异性的研究.

关键词＼cjkstart新粒子生成; 颗粒物增长; 转化机制; 环境效应; 大气复合污染
\end{abstract}

\section{Mechanism of New Particle Formation and Growth as well as Environmental Effects under Complex Air Pollution in China}

\author{
$\mathrm{Hu}, \mathrm{Min}^{*} \quad$ Shang, Dongjie Guo, Song Wu, Zhijun \\ (State Key Joint Laboratory of Environmental Simulation and Pollution Control, College of Environmental Sciences and \\ Engineering, Peking University, Beijing 100871)
}

\begin{abstract}
New particle formation (NPF) and its subsequent growth plays a key role in air quality and climate change at regional and global scales. Especially under complex air pollution in China, nucleation and growth can be highly efficient, claimed to be a main source of cloud condensation nuclei $(\mathrm{CCN})$ and an important cause of secondary aerosol pollution. Currently, the mechanism of particle formation and growth as well as its environmental effects are still poorly understood. Thereby, fully understanding of the atmospheric nucleation and subsequent growth still presents a big challenge to atmospheric chemistry researches. This study reviews the current results from studies on mechanisms and environmental effects of atmospheric nucleation and growth. We summarize that traditional nucleation theories such as binary nucleation of $\mathrm{H}_{2} \mathrm{SO}_{4}-\mathrm{H}_{2} \mathrm{O}$, ternary nucleation of $\mathrm{H}_{2} \mathrm{SO}_{4}-\mathrm{NH}_{3}-\mathrm{H}_{2} \mathrm{O}$, ion-induced nucleation are not capable in explaining new particle formation under complex air pollution, while newly proposed mechanisms such as organic acids and amine induced nucleation were not verified because of technique limitation. We propose that the future researches should focus on identifying the key chemical precursor response for driving nucleation and initial and subsequent growth, and understand the physical and chemical processing of new particle formation and growth. In particularly, application and development of novel techniques, such as APi-TOF-CIMS, PSM, Nano-HTDMA in new particle formation study is very important. Also, future researches should establish whole process tracking on new particle formation, from precursor, nucleation, growth till the environmental effects, by integrating field observation, chamber simulation, and modelling. Currently, the mechanism of highly efficient nucleation and rapid growth taking place under complex air pollution in China is urgently needed to be in-depth studied in order to improve our understanding of regional haze formation. This could be helpful to understand the similarity and difference in the nucleation mechanism between clean and polluted atmospheric environments.
\end{abstract}

Keywords new particle formation; particle growth; transformation mechanism; environmental impact; complex air pollution

\section{1 引言}

新粒子生成(New Particle Formation, NPF)与增长是 大气中重要的气态向颗粒相的二次转化过程, 影响人体 健康、区域环境质量和全球气候 ${ }^{[1,2]}$. 新粒子生成在全球
尺度上是大气颗粒物的重要来源之一; 在我国，新粒子 生成产生的高数浓度纳米颗粒物在高浓度气态污染物 和强氧化性背景下, 可以在几小时内快速增长至几十甚 至上百纳米 ${ }^{[3]}$, 一方面在大气中贡献高数浓度的云凝结

*E-mail: minhu@pku.edu.cn; Tel.: 010-62759880

Received February 26, 2016; published March 23, 2016.

Project supported by the National Natural Science Foundation of China (Nos. 91544214, 21190052, 41121004), National Basic Research Program of China (No. 2013CB228503) and Special Fund for Strategic Pilot Technology Chinese Academy of Sciences (No. XDB05010500).

项目受国家自然科学基金(Nos. 91544214, 21190052, 41121004), 国家重点基础研究发展计划(No. 2013CB228503)和中国科学院战略性先导科技专项 (No. XDB05010500)资助. 
核 $(\mathrm{CCN})$, 改变云物理过程和全球辐射平衡 ${ }^{[4]}$; 另一方 面也使得 $\mathrm{PM}_{2.5}$ 在若干天内增长数百微克每立方米, 是 我国严重的二次颗粒物污染和雽的重要诱因 ${ }^{[5]}$. 新粒生 成的超细颗粒物也有明显的人体健康影响, 其在人体内 的沉积能力较强, 可通过破坏细胞膜、生成自由基等诱 发哮喘、癌症等疾病 ${ }^{[6]}$. 所以, 揭示新粒子生成中气态污 染物向颗粒态转化的化学机制, 有效评估新粒子生成在 区域乃至全球范围内的环境、健康效应, 对于大气环境 科学研究有着重要意义. 本文阐述了新粒子生成研究中 重点关注的科学问题, 总结了国内外已有研究在本领域 所取得的进展和存在的不足, 并对未来我国开展新粒子 生成研究工作提出了展望, 以便于研究学者能够克服当 前新粒子研究的局限性, 进一步完善对于我国新粒子生 成的化学机制和环境影响的认识.

\section{2 新粒子生成研究主要进展}

新粒子生成可以分为成核和增长两个阶段, 成核是 指通过气相-液相一固相转化过程形成热力学稳定的 1 $\mathrm{nm}$ 大小的分子簇, 而增长则划分为分子簇通过成核蒸 汽的冷凝、非均相成核等增长至 $3 \mathrm{~nm}$ 的初始增长过程, 以及通过碰并和蒸汽冷凝等增长为更大尺寸的后续增 长过程 ${ }^{[7]}$. 针对新粒子生成的研究, 最关注的问题主要 包括: (1)新粒子成核机制, 即何种气态物质可以参与成 核, 具体化学途径如何; (2)新粒子发生的限制因素, 即 何种前体物浓度和凝结汇条件下, 新粒子可以发生; (3) 新粒子增长机制, 即各种气态前体物对于新粒子初始增 长和后续增长的贡献如何; (4)新粒子的区域环境效应, 即新粒子在污染条件下如何持续增长至积聚模态, 及其 对 $\mathrm{CCN}$ 和霧污染的贡献如何. 虽然在新粒子生成各个 过程的化学、物理机制方面取得了一定的进展, 但是直 于研究手段的局限性, 仍存在不清楚的问题.

\section{1 新粒子生成和增长机制及其环境影响}

针对成核机制, 研究普遍认为气态硫酸是参与成核 过程的关键前体物, 然而传统的硫酸一水二元均相成核 理论, 以及基于气态硫酸和成核速率的定量关系的活化 成核和动力学成核理论可以解释较低的新粒子成核速 率, 但不能全面解释高成核速率的新粒子生成事件 ${ }^{[8,9]}$. 因而可以降低气态硫酸饱和蒸汽压、促进成核的物质被

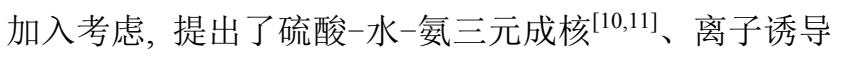
成核 ${ }^{[12]}$ 、碘参与成核 ${ }^{[13,14]}$ 等理论. 这些理论得到了一定 外场观测以及实验室模拟结果的支持, 但仍无法完全解 释我国大气较高的成核速率 ${ }^{[15]}$. 而近年来, 有机酸、有 机胺参与的热动力学成核被认为是解释高成核速率的 一种途径 ${ }^{[7]}$. 烟雾箱研究表明, 在硫酸-水的反应体系中 加入有机胺、芳香酸或蒎酮酸(通过加入单萜烯氧化得 到), 可以显著提升硫酸的成核能力, 达到与大气中成 核速率相匹配的水平 ${ }^{[16 ~ 18]}$. 而外场观测也发现了大气 有机酸含量和成核速率之间显著相关 ${ }^{[19]}$. 在模型模拟
方面, Paasonen 等 ${ }^{[20]}$ 和 Wang 等 ${ }^{[21]}$ 分别将可能参与颗粒 物增长的有机物浓度和模拟所得挥发性有机物(VOCs) 氧化产物浓度作为成核参数加入动力学成核模型中, 发 现可以较好地重现大气中的成核过程. 另外, 在成核发 生的限制因素方面，目前认为当大气中气态硫酸等前体 物达到一定浓度即可发生成核过程 ${ }^{[22]}$, 而已存在高浓 度颗粒物作为凝结汇和碰并汇将抑制新粒子生成. 然 而, 已存在颗粒物的数浓度、表面积浓度、质量浓度等 哪一个参数对抑制新粒子生成的影响更大，则存在争 议. 新粒子生成时源、汇的总体特征如何，仍然缺乏基 于长期观测得出的统计分析抑或实验模拟结果. 研究成 核机制的关键点和难点在于高分辨率、高灵敏度的大气 中气态前体物测量, 以及 $1 \sim 10 \mathrm{~nm}$ 颗粒物数浓度测量, 进而验证所提出理论的可靠性. 近年来, 国际已有先进 的测量仪器如下: 常压化学电离化飞行时间质谱 (APi-ToF-CIMS) 可以测量获得大气中气态硫酸、有机胺 的分子信息; 高灵敏度激光诱导荧光(LIF) 可测量大气 $\mathrm{OH}$ 自由基浓度进而用以估算气态硫酸，而颗粒物粒径 扩增器(PSM)、纳米凝结核计数器(Nano-CPC)和中性粒 子/离子谱仪(NAIS)则可以在线测量低至 $1 \mathrm{~nm}$ 左右的颗 粒物与离子数谱 ${ }^{[23]}$. 总而言之, 气态硫酸是成核的关键 物种, 而有机物等在成核中的作用是当前以及未来的研 究热点.

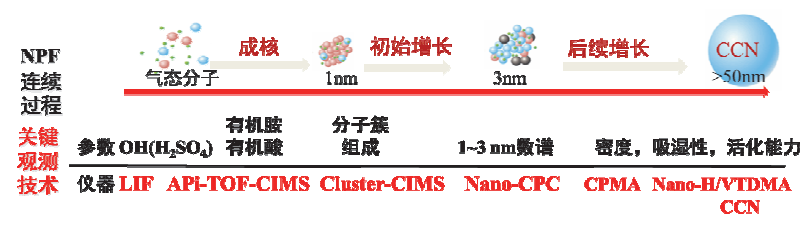

图 1 新粒子生成与增长及气候效应研究过程中的关键技术

Figure 1 Key techniques in research of new particle formation and growth as well as environmental effects

关于新粒子增长机制, 新粒子增长包括 $1 \sim 3 \mathrm{~nm}$ 的 初始增长和从 $3 \mathrm{~nm}$ 增长至几十甚至上百纳米的后续增 长过程 ${ }^{[24]}$. 新粒子增长过程是链接气态分子和具有显 著光学、气候效应尺寸的颗粒物之间的桥梁. 无论是对 气候效应还是对区域空气质量和健康的影响，新粒子的 增长过程都起到决定性作用. 由于成核后的新粒子只有 几纳米，虽然理论上当粒径大于临界粒径会自发增长， 但是受开尔文效应的影响使得新粒子初始增长不能通 过碰并和凝结过程简单的解释, 而且参与增长的物种需 要具有非常低的蒸汽压, 因此为研究新粒子增长带来较 大挑战.

对于新粒子增长机制的研究, 目前认为新粒子初始 增长机制可能包括成核蒸汽凝结、气态物质非均相反 应、离子诱导的分子簇形成增长 ${ }^{[7]}$. 已有的研究结论是 有机物在新粒子初始增长过程中, 起到重要的作用 ${ }^{[25]}$. 常见的生物源和人为源 VOCs 的氧化产物对新粒子的增 
长都具有重要作用, 虽然异戊二烯对新粒子生成具有抑 制作用 ${ }^{[26]}$, 但是异戊二烯的氧化产物已经被证明可以 参与到新粒子增长过程中, 考虑到在全球范围内异龙二 烯的排放量, 其氧化产物对新粒子增长的贡献不可忽 略 ${ }^{[27]}$. 最近的研究表明, 单萜烯氧化生成的极低挥发性 有机中间产物(ELVOCs) 可以有效促进新粒子的增长过 程 ${ }^{[28]}$, 而有机聚合物(Polymer)由于具有极低的挥发性, 因此非常有可能对新粒子的增长具有重要贡献 ${ }^{[29]}$. 已 有一定的外场、实验室研究表明, 二次有机组分在我国 大气颗粒物中占有较大比重, 因而定量二次有机物在颗 粒物增长中的作用, 是我国未来的新粒子增长研究的趋 势之一.

后续增长过程主要包括气态物质如有机物凝结、均 相反应以及颗粒物模态内的碰并. 气态硫酸、无机氨、 有机胺等物质可以通过形成硫酸盐、有机酸盐、有机胺 盐等参与颗粒物增长 ${ }^{[30 ~ 32]}$, 外场研究也印证了二次有 机组分在颗粒物中占到较大比重 ${ }^{[33,34]}$, 然而有机物贡献 后续增长的机制仍然存在诸多疑问 ${ }^{[25]}$, 需要同步测量 大气中核模态以及爱根模态颗粒物的理化特征和前体 物性质加以研究与验证. 目前可以在线测量超细颗粒 物、分子簇化学组成特征的仪器包括分子簇化学电离质 谱(Cluster-CIMS) 以及高分辨率飞行时间气溶胶质谱 (HR-ToF-AMS)等, 而纳米颗粒吸湿和挥发-串联电迁移 分析仪 (Nano-H/V-TDMA) 与离心颗粒物质量分析仪 (CPMA) 则可通过测量核模态颗粒物密度、吸湿、挥发 性推测其化学组成.

新粒子生成对气候效应有着显著地影响. 新粒子生 成后会持续增长至云凝结核大小, 是云凝结核的重要来 源 ${ }^{[35]}$. 全球尺度, 新粒子贡献了 $5 \% \sim 50 \%$ 行星边界层 的 CCN 浓度(过饱和度 $1 \%)^{[36]}$. 作者团队的研究结果表 明我国城市地区新粒子的后续增长可以使 $\mathrm{CCN}$ 浓度升 高 $0.4 \sim 6$ 倍 ${ }^{[37]}$. Kazil 等 ${ }^{[38]}$ 使用 ECHAM5-HAM 模型对 于大气核化、增长形成凝结核, 以及云凝结核对于云物 理过程、反照率、降雨等的影响进行了全球尺度的模拟, 结果表明大气核化在陆地地区总体可以贡献 -0.37 $\mathrm{W} / \mathrm{m}^{2}$ 的辐射强迫, 而在海洋区域则高达 $-2.18 \mathrm{~W} / \mathrm{m}^{2}$.

新粒子生成也与二次颗粒物污染密切相关. 新粒子 生成不仅可以急剧提升颗粒物数浓度, 新生成的颗粒物 作为凝结核, 可以通过有机蒸汽凝结、碰并等途径增长, 提升颗粒物质量浓度、增强消光作用, 从而影响区域空 气质量. 尤其是在相对污染的大气中出现的新粒子生成 事件, 由于 $\mathrm{SO}_{2}$ 、挥发性有机物等气态前体物浓度较高, 使得可凝结蒸汽浓度偏高, 加之可碰并的新生成核模态 颗粒物水平较高, 新生成粒子可快速增长, 在一天至若 干天内可使得爱根模态、积聚模态颗粒物浓度提升, 是 颗粒物二次污染的重要诱因 ${ }^{[5]}$. 现阶段基于实测的新粒 子生成与增长的环境效应评估仍十分有限, 尤其是新粒 子对颗粒物二次污染影响机制的认识以及环境中新粒 子对 $\mathrm{CCN}$ 的贡献实测信息十分缺乏. 因此, 有必要基于
观测和模型模拟将新粒子生成和增长机制与其环境影 响相结合，精确评估新粒子对霧形成和区域气候的影 响.

\section{2 新粒子生成国内外研究进展与不足}

20 世纪末以来，国际上针对新粒子生成开展了几 个大项目研究, 包括 Biogenic Aerosol Formation over the Boreal Forests (芬兰 Hyytiälä 森林)、Formation and Fate in the Coastal Environment (爱尔兰 Mace Head 海岸 站点)、Quantification of Aerosol Nucleation in the European Boundary Layer (意大利 San Pietro 城市站点)、 European Integrated project on Aerosol Cloud Climate and Air Quality Interactions (欧盟 25 个国家)、Nucleation and Real Time Characterization Experiment (美国 Atlanta)以 及 Cosmics Leaving Outdoor Droplets (瑞士 CERN 研究 所)等. 虽然这些项目对于新粒子生成现象建立了初步 描述和解释, 不过其化学机制仍存在较大争议, 而且这 些项目建立的相关理论和模型，均是基于清洁大气所得 的观测数据, 其在发展中国家的适用性并未被广泛检 验. 尤其是我国大气复合污染条件下，新粒子生成在背 景颗粒物浓度较高时仍然可以频繁发生，其核化、增长 速率、最终可以增长到的尺寸以及造成的环境效应也明 显高于相对清洁的大气 ${ }^{[39,40]}$, 这一现象是否可以用国际 上清洁地区研究所得机制解释仍然存疑 ${ }^{[15]}$, 因而在我 国开展新粒子生成研究显得更为重要.

在国内研究方面, 我国已经在新粒子生成研究方面 开展了一定工作. 作者研究团队最早于 2004 年在北京 城市大气观测到新粒子生成，由此基于北京大学城市大 气环境定位观测站开展长期观测 ${ }^{[41,42]}$. 近十年以来, 在 华北、长三角、珠三角等城市群, 泰山、瓦里关等高山 区域, 以及东南沿海均观测到大气新粒子生成事 件 ${ }^{[43 \sim 51]}$, 推动了我国新粒子生成和增长机制的研究. 作 者研究团队揭示了北京地区存在 “污染型” 和 “清洁型” 两种不同气象因素和数谱特征的新粒子生成现象, 同时 新粒子增长也存在 “贫硫型” 和 “富硫型” 两种机制 ${ }^{[52]}$. $\mathrm{Yu}$ 等 ${ }^{[53 \sim 55]}$ 基于外场观测和模型模拟对于有机物参与成 核、增长以及离子诱导成核机制进行了大量细致研究; $\mathrm{Li}$ 等 ${ }^{[56]}$ 总结了离子参与的新粒子生成研究成果. Nie 等 ${ }^{[57]}$ 研究发现由于沙尘传输过程中与化石燃料燃烧排 放的人为污染物混合后, “污染” 的沙尘在光照条件下 发生非均相光化学催化反应, 产生额外的大气自由基, 将更多的二氧化硫氧化生成气态硫酸以及更多的高挥 发性有机物氧化成低挥发性有机物, 从而导致了快速的 新粒子生成和增长.

尽管新粒子生成的研究得到关注, 成为研究重点, 并且取得了一定成果, 但是已有的研究还存在以下不 足: (1)先进测量技术亟待推广. 上文中提到的有机酸、 有机胺等气态前体物浓度，核化直接生成的 $1 \mathrm{~nm}$ 颗粒 物数浓度, 纳米级颗粒物理化性质等关键参数的测量技 
术, 仅应用于少数发达国家的清洁地区, 在我国应用胗 零无几，这限制了已有核化、增长机制在我国污染大气 中的验证; (2)研究关注点较为片段化. 已有研究多是对 新粒子生成单一环节的研究, 缺乏从气态前体物一成核一 增长一理化特征-环境效应等多参数、全过程的综合观测, 因此，无法有效沟通起新粒子生成从气态前体物到环境 效应的演变全过程; (3)研究方法较为单一. 在研究方法 上结合外场观测、实验模拟和模型模拟的闭合研究在我 国污染地区也几乎没有开展. 基于我国特有的大气复合 污染背景, 建立一套沟通高前体物浓度、高凝结汇, 特 殊成核机制以及强环境效应的大气新粒子生成全过程 的研究方法, 并开展观测和模拟的协同研究, 是我国大 气化学研究亟待开展的工作.

\section{3 总结与展望}

随着近年来对新粒子、前体物及其环境效应相关测 定技术的不断涌现, 给基于对真实环境大气观测的新粒 子生成研究提供了更多的可行性. 面对以下 3 个方面的 挑战, 有可能形成突破.

(1)研究技术上, 缺少对新粒子生成长期的综合观 测, 尤其是气态前体物(硫酸、有机物)和增长后化学组 成等关键参数的测量.

(2)研究思路上, 缺乏从气态前体物一成核-增长-环 境效应等多参数、全过程综合观测, 以及将外场观测、 烟雾箱模拟和模型模拟相结合的综合研究.

(3)大气复合污染条件下, 我国大气污染过程复杂, 不同大气环境、不同时间存在着成核和增长机制差异.

我国高污染、强氧化性为特点的大气复合污染条件 下, 新粒子关注的主要科学问题是: (1)气态硫酸与有机 前体物在我国大气复合污染条件下的高效成核过程中 发挥怎样的作用? (2)大气复合污染条件下新粒子初始 增长和后续增长的物理化学机制如何? 区别于清洁地 区, 我国新粒子快速的后续增长的机制是什么? (3)我国 频繁高效的新粒子生成与持续快速增长对区域空气质 量乃至全球气候效应的影响是什么?

为全面理解大气复合污染条件下成核和增长机制 及其环境效应, 从而提高对区域皬形成机制的认识, 未 来新粒子生成领域主要研究方向和目标是:

(1)识别新粒子生成的关键前体物, 弄清新粒子生 成的决定因素. 完善精确测定关键气态前体物包括硫 酸、有机酸、有机胺的技术手段, 结合小至 $1 \mathrm{~nm}$ 的新粒 子数谱分布测定和模式模拟, 识别大气复合污染条件下 大气成核的关键前体物和成核机制，探究关键前体物协 同还是单一物种导致我国大气中新粒子的高成核速率. 通过先进的研究手段, 如环境大气烟雾箱, 揭示我国大
气复合污染条件下新粒子生成的决定因素.

(2)揭示粒径分辨的新粒子增长的物理化学过程. 通过追踪测定新粒子的物理、化学、热动力学性质, 表 征新粒子增长过程中物理化学性质变化, 弄清驱动新粒 子增长的物理化学机制及其与粒径的相互关系, 尤其是 从分子水平对纳米颗粒物的化学组成进行表征, 深入揭 示有机物在新粒子增长过程中的作用. 结合烟雾箱模拟 和理论模型，明晰不同粒径尺度段(模态下)新粒子增长 机制.

弄清驱动新粒子初始和后续增长的化学和物理机 制. 揭示不同化学物种如何通过凝结和化学反应导致新 粒子增长的化学过程, 认识新粒子生成和增长的动力学 过程以及挥发性和吸湿性等物理特性的演变.

(3)揭示新粒子高频率生成与快速持续增长并致䨪 的条件与机制. 结合外场综合观测和环境大气烟雾箱模 拟实验, 针对高频率新粒子生成并快速和持续增长最终 致䨪的典型事件进行深入研究，结合气象条件、痕量气 态污染物浓度、颗粒物物理化学组分, 辨识此类新粒子 事件的发生条件、链接新粒子致䨪的关键物理化学过程, 探讨快速增长并致霧的新粒子事件的增长机制与清洁 环境的差异.

揭示大气复合污染条件下快速成核和持续增长的 条件与机制. 认识在什么气象条件下，哪类前体物占主 导情况下, 新粒子可以持续并快速增长, 最终形成区域 性䨪. 识别出有利于新粒子快速成核和持续增长的气象 条件和前体物。

(4)评价新粒子生成与增长的大气环境影响. 评估 新粒子生成事件对区域细颗粒物 $\mathrm{PM}_{2.5}$ 质量增量与气候 相关因子的贡献. 基于外场观测和上述研究获得的成核 与增长机理, 采用盒子模型模拟新粒子成核与增长, 量 化新粒子生成事件对区域细颗粒物 $\mathrm{PM}_{2.5}$ 质量增量、对 消光以及气候相关因子 $\mathrm{CCN}$ 的贡献, 评估区域新粒子 发生与增长对我国大气复合污染形成的关键作用，重点 关注在怎样的大气复合污染和气象条件下，新粒子可以 快速持续增长到积聚模态，直接影响到大气环境质量和 增加云凝结核数量, 探讨新粒子在霧形成中的物理和化 学机制. 进而, 为制定我国区域颗粒物污染控制政策提 供科学依据, 为降低新粒子全球气候效应模型的不确定 性提供污染地区的案例.

\section{致谢}

感谢北京大学大气化学团队和德国 Leibniz Institute for Tropospheric Research A. Wiedensohler 教授和美国 Texas A\&M University 张人一教授长期的合作与支持. 


\section{作者简介}

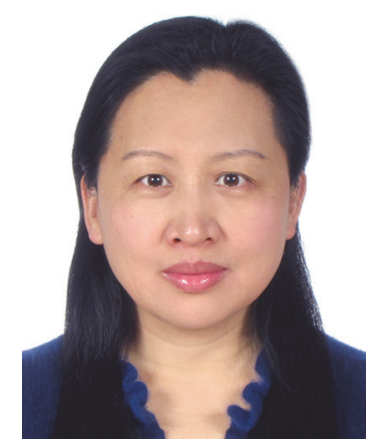

胡敏, 北京大学环境科学与工程学院教授, 1987 年 毕业于北京大学应用化学学士学位, 1990 年毕业于北京 大学环境科学硕士学位, 1993 年毕业于北京大学环境科 学博士学位. 1993 年至今在北京大学工作. 研究方向大 气颗粒物来源转化及其环境影响.

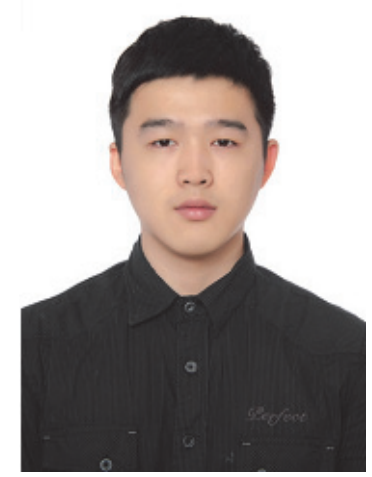

尚冬杰, 北京大学环境科学与工程学院博士生, 2013 年毕业于北京大学环境科学学士学位, 主要研究方 向为大气新粒子生成、增长机制及其环境效应. 参与的 课题包括环保公益性行业科研专项 “我国南部、西南部 空气污染物跨界输送及应对战略研究”与 “东亚地区 大气污染物跨界输送及其相互影响与应对策略研究”, 科技部 973 项目 “化石燃料燃烧排放 $\mathrm{PM}_{2.5}$ 源头控制技 术的基础研究”等.

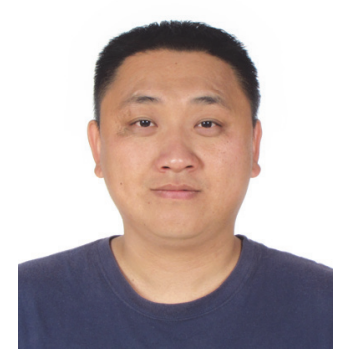

郭松, 北京大学环境科学与工程学院研究员, 2005 年毕业于北京大学环境学院获得学士学位, 2011 年获得 北京大学环境科学博士学位, 2015 年 7 月获得北京大学 助理教授/研究员职位, 2016 年入选中组部 “青年千人计
划”. 主要研究方向为大气颗粒物来源和二次转化机制 研究, 包括新粒子生成与增长、二次有机气溶胶组成和 黑碳颗粒物老化机制.

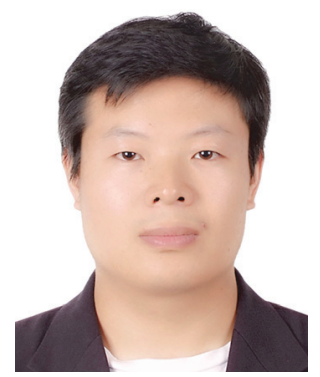

吴志军, 北京大学环境科学与工程学院研究员, 2001 年毕业于吉林大学获得学士学位, 2007 年毕业于北 京大学获得博士学位, 2012 年底入选中组部“青年千人 计划”。主要开展的研究工作包括不同大气环境, 尤其 是高污染、强氧化性大气中新粒子生成和增长机理; 大 气颗粒物与水相互作用.

\section{References}

[1] Kulmala, M. Science 2003, 302, 1000.

[2] Zhang, R. Science 2010, 328, 1366.

[3] Wiedensohler, A.; Birmili, W.; Nowak, A.; Sonntag, A.; Weinhold, K.; Merkel, M.; Wehner, B.; Tuch, T.; Pfeifer, S.; Fiebig, M.; Fjäraa, A. M.; Asmi, E.; Sellegri, K.; Depuy, R.; Venzac, H.; Villani, P.; Laj, P.; Aalto, P.; Ogren, J. A.; Swietlicki, E.; Williams, P.; Roldin, P.; Quincey, P.; Hüglin, C.; Fierz-Schmidhauser, R.; Gysel, M.; Weingartner, E.; Riccobono, F.; Santos, S.; Grüning, C.; Faloon, K.; Beddows, D.; Harrison, R.; Monahan, C.; Jennings, S. G.; O'Dowd, C. D.; Marinoni, A.; Horn, H. G.; Keck, L.; Jiang, J.; Scheckman, J.; McMurry, P. H.; Deng, Z.; Zhao, C. S.; Moerman, M.; Henzing, B.; de Leeuw, G.; Löschau, G.; Bastian, S. Atmos. Meas. Tech. 2012, 5 , 657.

[4] Carslaw, K. S.; Lee, L. A.; Reddington, C. L.; Pringle, K. J.; Rap, A.; Forster, P. M.; Mann, G. W.; Spracklen, D. V.; Woodhouse, M. T.; Regayre, L. A.; Pierce, J. R. Nature 2013, 503, 67.

[5] Guo, S.; Hu, M.; Zamora, M. L.; Peng, J.; Shang, D.; Zheng, J.; Du, Z.; Wu, Z.; Shao, M.; Zeng, L.; Molina, M. J.; Zhang, R. Proc. Natl. Acad. Sci. 2014, 111, 17373.

[6] Sioutas, C.; Delfino, R. J.; Singh, M. Environ. Health Perspect. 2005, 113, 947.

[7] Zhang, R.; Khalizov, A.; Wang, L.; Hu, M.; Xu, W. Chem. Rev. 2012, 112, 1957.

[8] Kulmala, M.; Lehtinen, K. E. J.; Laaksonen, A. Atmos. Chem. Phys. 2006, 6, 787.

[9] Wang, Z. B.; Hu, M.; Yue, D. L.; Zheng, J.; Zhang, R. Y.; Wiedensohler, A.; Wu, Z. J.; Nieminen, T.; Boy, M. Atmos. Chem. Phys. 2011, 11, 12663.

[10] Kulmala, M.; Pirjola, L.; Makela, J. M. Nature 2000, 404, 66.

[11] Kerminen, V. M.; Petäjä, T.; Manninen, H. E.; Paasonen, P.; Nieminen, T.; Sipilä, M.; Junninen, H.; Ehn, M.; Gagné, S.; Laakso, L.; Riipinen, I.; Vehkamäki, H.; Kurten, T.; Ortega, I. K.; Dal Maso, M.; Brus, D.; Hyvärinen, A.; Lihavainen, H.; Leppä, J.; Lehtinen, K. E. J.; Mirme, A.; Mirme, S.; Hõrrak, U.; Berndt, T.; Stratmann, F.; Birmili, W.; Wiedensohler, A.; Metzger, A.; Dommen, J.; Baltensperger, U.; Kiendler-Scharr, A.; Mentel, T. F.; Wildt, J.; Winkler, P. M.; Wagner, P. E.; Petzold, A.; Minikin, A.; Plass- Dülmer, C.; Pöschl, U.; Laaksonen, A.; Kulmala, M. Atmos. Chem. Phys. 2010, 10, 10829.

[12] Kirkby, J.; Curtius, J.; Almeida, J.; Dunne, E.; Duplissy, J.; Ehrhart, S.; Franchin, A.; Gagne, S.; Ickes, L.; Kurten, A.; Kupc, A.; Metzger, A.; Riccobono, F.; Rondo, L.; Schobesberger, S.; Tsagkogeorgas, G.; Wimmer, D.; Amorim, A.; Bianchi, F.; Breitenlechner, M.; David, A.; Dommen, J.; Downard, A.; Ehn, M.; Flagan, R. C.; Haider, S.; Hansel, A.; Hauser, D.; Jud, W.; Junninen, H.; Kreissl, 
F.; Kvashin, A.; Laaksonen, A.; Lehtipalo, K.; Lima, J.; Lovejoy, E. R.; Makhmutov, V.; Mathot, S.; Mikkila, J.; Minginette, P.; Mogo, S.; Nieminen, T.; Onnela, A.; Pereira, P.; Petaja, T.; Schnitzhofer, R.; Seinfeld, J. H.; Sipila, M.; Stozhkov, Y.; Stratmann, F.; Tome, A.; Vanhanen, J.; Viisanen, Y.; Vrtala, A.; Wagner, P. E.; Walther, H.; Weingartner, E.; Wex, H.; Winkler, P. M.; Carslaw, K. S.; Worsnop, D. R.; Baltensperger, U.; Kulmala, M. Nature 2011, 476, 429.

[13] O'Dowd, C. D. J. Geophys. Res. 2001, 106, 1545.

[14] Allan, J. D.; Williams, P. I.; Najera, J.; Whitehead, J. D.; Flynn, M. J.; Taylor, J. W.; Liu, D.; Darbyshire, E.; Carpenter, L. J.; Chance, R.; Andrews, S. J.; Hackenberg, S. C.; McFiggans, G. Atmos. Chem. Phys. 2015, 15, 5599.

[15] Wang, Z.; Hu, M.; Wu, Z.; Yue, D. Acta Chim. Sinica 2013, 71, 519. (王志斌, 胡敏, 吴志军, 岳玎利, 化学学报, 2013, 71, 519.)

[16] Zhang, R.; Suh, I.; Zhao, J.; Zhang, D.; Fortner, E. C.; Tie, X.; Molina, L. T.; Molina, M. J. Science 2004, 304, 1487.

[17] Almeida, J.; Schobesberger, S.; Kurten, A.; Ortega, I. K.; Kupiainen-Maatta, O.; Praplan, A. P.; Adamov, A.; Amorim, A.; Bianchi, F.; Breitenlechner, M.; David, A.; Dommen, J.; Donahue, N. M.; Downard, A.; Dunne, E.; Duplissy, J.; Ehrhart, S.; Flagan, R. C.; Franchin, A.; Guida, R.; Hakala, J.; Hansel, A.; Heinritzi, M.; Henschel, H.; Jokinen, T.; Junninen, H.; Kajos, M.; Kangasluoma, J.; Keskinen, H.; Kupc, A.; Kurten, T.; Kvashin, A. N.; Laaksonen, A.; Lehtipalo, K.; Leiminger, M.; Leppa, J.; Loukonen, V.; Makhmutov, V.; Mathot, S.; McGrath, M. J.; Nieminen, T.; Olenius, T.; Onnela, A.; Petaja, T.; Riccobono, F.; Riipinen, I.; Rissanen, M.; Rondo, L.; Ruuskanen, T.; Santos, F. D.; Sarnela, N.; Schallhart, S.; Schnitzhofer, R.; Seinfeld, J. H.; Simon, M.; Sipila, M.; Stozhkov, Y.; Stratmann, F.; Tome, A.; Trostl, J.; Tsagkogeorgas, G.; Vaattovaara, P.; Viisanen, Y.; Virtanen, A.; Vrtala, A.; Wagner, P. E.; Weingartner, E.; Wex, H.; Williamson, C.; Wimmer, D.; Ye, P.; Yli-Juuti, T.; Carslaw, K. S.; Kulmala, M.; Curtius, J.; Baltensperger, U.; Worsnop, D. R.; Vehkamaki, H.; Kirkby, J. Nature 2013, $502,359$.

[18] Riccobono, F.; Schobesberger, S.; Scott, C. E.; Dommen, J.; Ortega, I. K.; Rondo, L.; Almeida, J.; Amorim, A.; Bianchi, F.; Breitenlechner, M.; David, A.; Downard, A.; Dunne, E. M.; Duplissy, J.; Ehrhart, S.; Flagan, R. C.; Franchin, A.; Hansel, A.; Junninen, H.; Kajos, M.; Keskinen, H.; Kupc, A.; Kürten, A.; Kvashin, A. N.; Laaksonen, A.; Lehtipalo, K.; Makhmutov, V.; Mathot, S.; Nieminen, T.; Onnela, A.; Petäjä, T.; Praplan, A. P.; Santos, F. D.; Schallhart, S.; Seinfeld, J. H.; Sipilä, M.; Spracklen, D. V.; Stozhkov, Y.; Stratmann, F.; Tomé, A.; Tsagkogeorgas, G.; Vaattovaara, P.; Viisanen, Y.; Vrtala, A.; Wagner, P. E.; Weingartner, E.; Wex, H.; Wimmer, D.; Carslaw, K. S.; Curtius, J.; Donahue, N. M.; Kirkby, J.; Kulmala, M.; Worsnop, D. R.; Baltensperger, U. Science 2014, 344, 717.

[19] Kulmala, M.; Kontkanen, J.; Junninen, H.; Lehtipalo, K.; Manninen, H. E.; Nieminen, T.; Petäjä, T.; Sipilä, M.; Schobesberger, S.; Rantala, P.; Franchin, A.; Jokinen, T.; Järvinen, E.; Äijälä, M.; Kangasluoma, J.; Hakala, J.; Aalto, P. P.; Paasonen, P.; Mikkilä, J.; Vanhanen, J.; Aalto, J.; Hakola, H.; Makkonen, U.; Ruuskanen, T.; Mauldin, R. L.; Duplissy, J.; Vehkamäki, H.; Bäck, J.; Kortelainen, A.; Riipinen, I.; Kurtén, T.; Johnston, M. V.; Smith, J. N.; Ehn, M.; Mentel, T. F.; Lehtinen, K. E. J.; Laaksonen, A.; Kerminen, V.-M.; Worsnop, D. R. Science 2013, 339, 943.

[20] Paasonen, P.; Olenius, T.; Kupiainen, O.; Kurtén, T.; Petäjä, T.; Birmili, W.; Hamed, A.; Hu, M.; Huey, L. G.; Plass-Duelmer, C.; Smith, J. N.; Wiedensohler, A.; Loukonen, V.; McGrath, M. J.; Ortega, I. K.; Laaksonen, A.; Vehkamäki, H.; Kerminen, V. M.; Kulmala, M. Atmos. Chem. Phys. 2012, 12, 9113.

[21] Wang, Z. B.; Hu, M.; Mogensen, D.; Yue, D. L.; Zheng, J.; Zhang, R. Y.; Liu, Y.; Yuan, B.; Li, X.; Shao, M.; Zhou, L.; Wu, Z. J.; Wiedensohler, A.; Boy, M. Atmos. Chem. Phys. 2013, 13, 11157.

[22] Sipilä, M.; Berndt, T.; Petäjä, T.; Brus, D.; Vanhanen, J.; Stratmann, F.; Patokoski, J.; Mauldin, R. L.; Hyvärinen, A.-P.; Lihavainen, H.; Kulmala, M. Science 2010, 327, 1243.

[23] Zhang, R.; Wang, G.; Guo, S.; Zamora, M. L.; Ying, Q.; Lin, Y.; Wang, W.; Hu, M.; Wang, Y. Chem. Rev. 2015, 115, 3803.

[24] Kulmala, M.; Kerminen, V.-M. Atmos. Res. 2008, 90, 132.

[25] Riipinen, I.; Yli-Juuti, T.; Pierce, J. R.; Petäjä, T.; Worsnop, D. R.; Kulmala, M.; Donahue, N. M. Nat. Geosci. 2012, 5, 453.

[26] Kiendler-Scharr, A.; Wildt, J.; Dal Maso, M.; Hohaus, T.; Kleist, E.; Mentel, T. F.; Tillmann, R.; Uerlings, R.; Schurr, U.; Wahner, A.
Nature 2009, 461, 381.

[27] Xu, W.; Gomez-Hernandez, M.; Guo, S.; Secrest, J.; Marrero-Ortiz, W.; Zhang, A. L.; Zhang, R. J. Am. Chem. Soc. 2014, 136, 15477.

[28] Ehn, M.; Thornton, J. A.; Kleist, E.; Sipilä, M.; Junninen, H.; Pullinen, I.; Springer, M.; Rubach, F.; Tillmann, R.; Lee, B.; Lopez-Hilfiker, F.; Andres, S.; Acir, I.-H.; Rissanen, M.; Jokinen, T.; Schobesberger, S.; Kangasluoma, J.; Kontkanen, J.; Nieminen, T.; Kurtén, T.; Nielsen, L. B.; Jørgensen, S.; Kjaergaard, H. G.; Canagaratna, M.; Maso, M. D.; Berndt, T.; Petäjä, T.; Wahner, A.; Kerminen, V.-M.; Kulmala, M.; Worsnop, D. R.; Wildt, J.; Mentel, T. F. Nature 2014, 506, 476.

[29] Wang, L.; Khalizov, A. F.; Zheng, J.; Xu, W.; Ma, Y.; Lal, V.; Zhang, R. Nat. Geosci. 2010, 3, 238.

[30] Dusek, U.; Frank, G. P.; Curtius, J.; Drewnick, F.; Schneider, J.; Kürten, A.; Rose, D.; Andreae, M. O.; Borrmann, S.; Pöschl, U. Geophys. Res. Lett. 2010, 37.

[31] Smith, J. N.; Dunn, M. J.; VanReken, T. M.; Iida, K.; Stolzenburg, M. R.; McMurry, P. H.; Huey, L. G. Geophys. Res. Lett. 2008, 35, 228.

[32] Bzdek, B. R.; Zordan, C. A.; Luther, G. W.; Johnston, M. V. Aerosol Sci. Technol. 2011, 45, 1041.

[33] Guo, S.; Hu, M.; Guo, Q.; Shang, D. Acta Chim. Sinica 2014, 72, 658. (郭松, 胡敏, 郭庆丰, 尚冬杰, 化学学报, 2014, 72, 658.)

[34] Guo, S.; Hu, M.; Shang, D.; Guo, Q.; Hu, W. Acta Chim. Sinica 2014, 72, 145. (郭松, 胡敏, 尚冬杰, 郭庆丰, 胡伟伟, 化学学报, 2014, 72, 145.)

[35] Kerminen, V. M.; Paramonov, M.; Anttila, T.; Riipinen, I.; Fountoukis, C.; Korhonen, H.; Asmi, E.; Laakso, L.; Lihavainen, H.; Swietlicki, E.; Svenningsson, B.; Asmi, A.; Pandis, S. N.; Kulmala, M.; Petäjä, T. Atmos. Chem. Phys. 2012, 12, 12037.

[36] Spracklen, D. V.; Carslaw, K. S.; Kulmala, M.; Kerminen, V.-M.; Sihto, S.-L.; Riipinen, I.; Merikanto, J.; Mann, G. W.; Chipperfield M. P.; Wiedensohler, A.; Birmili, W.; Lihavainen, H. Geophys. Res. Lett. 2008, 35, 160.

[37] Yue, D. L.; Hu, M.; Zhang, R. Y.; Wu, Z. J.; Su, H.; Wang, Z. B.; Peng, J. F.; He, L. Y.; Huang, X. F.; Gong, Y. G.; Wiedensohler, A. Atmos. Environ. 2011, 45, 6070.

[38] Kazil, J.; Stier, P.; Zhang, K.; Quaas, J.; Kinne, S.; O'Donnell, D.; Rast, S.; Esch, M.; Ferrachat, S.; Lohmann, U.; Feichter, J. Atmos. Chem. Phys. 2010, 10, 10733.

[39] Peng, J. F.; Hu, M.; Wang, Z. B.; Huang, X. F.; Kumar, P.; Wu, Z. J.; Guo, S.; Yue, D. L.; Shang, D. J.; Zheng, Z.; He, L. Y. Atmos. Chem. Phys. 2014, 14, 10249

[40] Zhu, Y.; Sabaliauskas, K.; Liu, X.; Meng, H.; Gao, H.; Jeong, C.-H.; Evans, G. J.; Yao, X. Atmos. Environ. 2014, 98, 655.

[41] Wehner, B.; Wiedensohler, A.; Tuch, T. M.; Wu, Z. J.; Hu, M.; Slanina, J.; Kiang, C. S. Geophys. Res. Lett. 2004, 31, 217.

[42] Wu, Z.; Hu, M.; Liu, S.; Wehner, B.; Bauer, S.; Ma ßling, A.; Wiedensohler, A.; Petäjä, T.; Dal Maso, M.; Kulmala, M. J. Geophys. Res. 2007, 112, D09209.

[43] Leng, C.; Zhang, Q.; Tao, J.; Zhang, H.; Zhang, D.; Xu, C.; Li, X.; Kong, L.; Cheng, T.; Zhang, R.; Yang, X.; Chen, J.; Qiao, L.; Lou, S.; Wang, H.; Chen, C. Atmos. Chem. Phys. 2014, 14, 11353.

[44] Wang, Z. B.; Hu, M.; Sun, J. Y.; Wu, Z. J.; Yue, D. L.; Shen, X. J.; Zhang, Y. M.; Pei, X. Y.; Cheng, Y. F.; Wiedensohler, A. Atmos. Chem. Phys. 2013, 13, 12495.

[45] Wang, H.; Zhu, B.; Shen, L.; An, J.; Yin, Y.; Kang, H. Atmos. Res. 2014, 150, 42 .

[46] Lin, P.; Hu, M.; Wu, Z.; Niu, Y.; Zhu, T. Atmos. Environ. 2007, 41, 6784.

[47] Gong, Y.; Su, H.; Cheng, Y.; Liu, F.; Wu, Z.; Hu, M.; Zeng, L.; Zhang, Y. Adv. Atmos. Sci. 2008, 25, 427.

[48] Yue, D. L.; Hu, M.; Wang, Z. B.; Wen, M. T.; Guo, S.; Zhong, L. J.; Wiedensohler, A.; Zhang, Y. H. Atmos. Environ. 2013, 76, 181.

[49] Kivekäs, N.; Sun, J.; Zhan, M.; Kerminen, V. M.; Hyvärinen, A.; Komppula, M.; Viisanen, Y.; Hong, N.; Zhang, Y.; Kulmala, M.; Zhang, X. C.; Deli, G.; Lihavainen, H. Atmos. Chem. Phys. 2009, 9, 5461.

[50] Shen, X. J.; Sun, J. Y.; Zhang, Y. M.; Wehner, B.; Nowak, A.; Tuch, T.; Zhang, X. C.; Wang, T. T.; Zhou, H. G.; Zhang, X. L.; Dong, F.; Birmili, W.; Wiedensohler, A. Atmos. Chem. Phys. 2011, 11, 1565.

[51] Chen, C.; Hu, M.; Wu, Z. J.; Wu, Y. S.; Guo, S.; Chen, W. T.; Luo, B.; Shao, M.; Zhang, Y. H.; Xie, S. D. China Environ. Sci. 2014, 34 2764. (陈晨, 胡敏, 吴志军, 吴宇声, 郭松, 陈文泰, 罗涁, 邵敏, 张远航, 谢绍东, 中国环境科学, 2014, 34, 2764.) 
[52] Yue, D. L.; Hu, M.; Zhang, R. Y.; Wang, Z. B.; Zheng, J.; Wu, Z. J.; Wiedensohler, A.; He, L. Y.; Huang, X. F.; Zhu, T. Atmos. Chem. Phys. 2010, 10, 4953.

[53] Yu, F.; Hallar, A. G. J. Geophys. Res.-Atmos. 2014, 119, 12

[54] Yu, F. J. Geophys. Res. 2010, 115, D03206.

[55] Yu, F.; Luo, G.; Liu, X.; Easter, R. C.; Ma, X.; Ghan, S. J. Atmos.
Chem. Phys. 2012, 12, 11451

[56] Li, Q.; Jiang, J.; Hao, J. KONA Powder Part. J. 2015, 32, 57.

[57] Nie, W.; Ding, A.; Wang, T.; Kerminen, V. M.; George, C.; Xue, L.; Wang, W.; Zhang, Q.; Petaja, T.; Qi, X.; Gao, X.; Wang, X.; Yang, X.; Fu, C.; Kulmala, M. Sci. Rep. 2014, 4, 6634. 